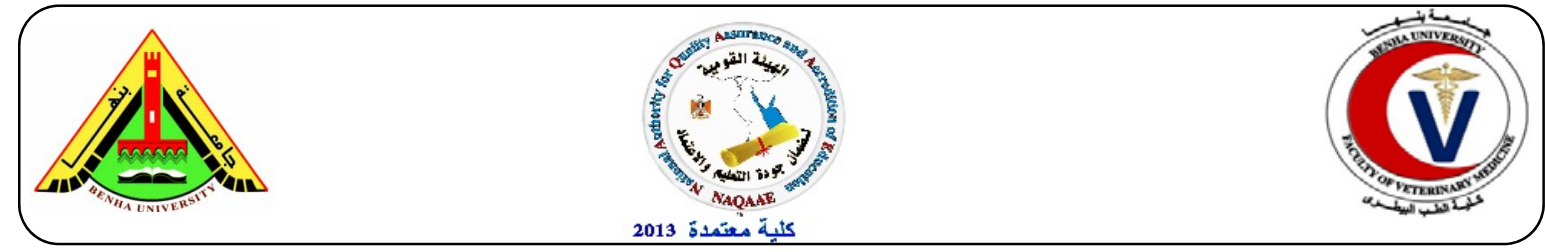

\title{
Generating LacZ-reporter transgenic mice to identify $\alpha 1$ (XIX) Collagen (Col19a1) expression in Dermal Papilla Cells
}

\author{
Ahmed Abo-Ahmed ${ }^{1,2}$, Dong-soo Lee ${ }^{1}$, Foad Farrag ${ }^{3}$, Xujun Han ${ }^{1}$, Md Nojebuzzaman', Mohamed \\ Attia $^{2}$, Hatem Bahgat ${ }^{2}$, Ahmed Kassab ${ }^{2}$, Masatake Osawa ${ }^{1, *}$ \\ ${ }^{1}$ Laboratory of Molecular Design and Synthesis, Department of Regenerative Medicine and Applied \\ Medical Sciences, Graduate School of Medicine, Gifu University, 1-1 Yanagido, Gifu 501-1194, Japan. \\ 2 Department of Anatomy and Embryology, Faculty of Veterinary Medicine, Benha University, \\ Moshtohor, Toukh 13736, Egypt. ${ }^{3}$ Department of Anatomy and Embryology, Faculty of Veterinary \\ Medicine, Kafr Elsheikh University, Kafr Elsheikh 33516, Egypt \\ *Author to whom correspondence should be addressed; E-Mail: masaosaw@gifu-u.ac.jp,
}

\section{A B S T R A C T}

It is widely accepted the concept that tissue morphogenesis is mediated by reciprocal interactions between epithelial and mesenchymal cells, whereas the molecular details of these interactions remain largely elusive. The hair follicle (HF) is a mini-organ whose proper morphogenesis is governed by a series of interactions between epidermal and dermal cells. Due to its relatively simple structure, the HF affords an excellent model to decipher the molecular mechanisms of epithelial - mesenchymal interactions. To explore the molecular mechanisms by which Dermal Papilla (DP) cells regulate hair follicle formation, we performed a comparative transcriptome analysis and identified numerous genes preferentially expressed in the DP cells. Among these, a gene encoding type XIX Collagen (Col19a1) has become the focus of our attention because of its extensive evolutionary conservation. We generated Col19a1 ${ }^{\text {LacZ }}$ reporter mice using CRISPR/Cas9 system to induce homologous recombination to recapitulate Col19a1 expression pattern. In mouse embryos, Col19a $1^{\text {LacZ }}$ expression is confined to the DP cells of hair follicles, ring sinus of whiskers, skeletal muscles and basal keratinocyte layer of skin epidermis of limbs and tail. Moreover, Col19 ${ }^{L a c Z}$ started to be expressed at E14.5 in the dermal condensates of hair germ stage to the DP cells of mature hair follicle but not in the placode stage. Ongoing generation of Col19a1 null mice will ultimately explore the functional role of Col19a1 during HF morphogenesis. From this perspective, it is plausible to expect that future studies will provide additional functional evidence for Col19a1 during development which might be a useful tool to understand the molecular mechanisms undergoing HF stem cell regulation.

Keywords: Mesenchymal, Morphogenesis, Col19a1, Dermal papilla, CRISPR/Cas9, Evolutionary. 


\section{INTRODUCTION}

Reciprocal interactions between epithelium and mesenchyme are common features of early stages of morphogenesis in different tissues and organs (Ribatti and Santoiemma, 2014). Embryonic hair follicle induction and formation are orchestrated by mesenchymal-epithelial interactions between specialized mesenchymal dermal (DP) cells and epidermal stem cells that switch to a hair fate (Sennett and Rendl, 2012). The Extracellular Matrix (ECM) consists of a highly heterogeneous mixture of interacting proteins that form a complex array of supra molecular structures, and that bind cell surface receptors and soluble signaling molecules (Ramirez and Rifkin, 2003). The collagens represent the largest family of structural ECM components with 27 designated trimers that are broadly divided into fibrillar and non-fibrillar collagen types (Myllyharju and Kivirikko, 2004). By our previous comparative transcriptome profiling using Hes5-EGFP transgenic mice, we identified genes which are preferentially expressed in DP cells. Among these genes, $\alpha 1$ Collagen XIX (Col19a1) which is specifically expressed in DP cells.

Collagen XIX (Col19) is a poorly characterized member of the fibrilassociated collagens with an interrupted triple helices (FACIT) class of collagen molecules. The Col19a1 gene maps to the q12-q14 region of human chromosome 6, the same region as the gene coding for the Col9a1 while the mouse Col19a1 gene is located in region A3 of chromosome 1, where the mouse Col9a1 gene has also been mapped. Taken together, this suggests that Col19a1 and Col9a1 were duplicated from the same ancestor gene of the FACIT family (Khaleduzzaman et al., 1997).

Col19a1 is an evolutionary conserved gene from Caenorhabditis elegans (C. elegans) to human. It is localized in the circumferential annular rings and the lateral trilaminar alae of the cuticle playing a fundamental role in C. elegans exoskeleton or cuticle morphogenesis (Thein et al., 2003). Type XIX collagen transcripts have been detected at very low levels in mouse embryonic tissues by the RT-PCR technique and in human rhabdomyosarcoma cell lines by Northern analysis (Myers et al., 1994; Sumiyoshi et al., 1997). Col19a1 expression during embryogenesis commences at E9.5 in the myotome and with a pattern that closely follows the myogenic regulatory factor myf5. Transient expression of Col19a1 in muscular tissues is confined to few sites of the developing embryo, such as limbs, tongue, and the smooth muscle layers of the stomach and esophagus. Additional nonmuscular sites of Col19a1 activity include the skin of the E16.5 embryos and the cerebral cortex and hippocampus of the new born brain (Sumiyoshi et al., 2001). Col19 is deposited at extremely low amounts in the basement membrane zones (BMZs) of vascular, neural, and mesenchymal tissues (Myers et al., 1997). Col19 forms higher order aggregates that may conceivably modulate cell-matrix interactions, cell-cell communications, and/or local 
concentrations of signaling molecules (Myers et al., 2003).

More interestingly, plasmin, one of the most important enzymes involved in tumor invasion, is able to release a fragment of $\mathrm{NC1}$ domain of Col19, which inhibits the migration capacities of tumor cells and exerts a strong inhibition of tumor growth (Oudart et al., 2015).

Aside from the rhabdomyosarcoma cell line, there is currently no information about the tissue distribution of type XIX collagen and, consequently, about its possible function. The present study was designed to generate nuclear LacZ (nLacZ) reporter transgenic mice to recapitulate and characterize the expression pattern of Col19a1 during mouse embryogenesis, in addition to providing useful information for the future functional analysis studies and characterization of type XIX collagen (Col19a1) null mice to elucidate its function.

\section{MATERIAL AND METHODS}

\subsection{Animals}

Animal handling procedures were approved by the Committee for Animal Research and Welfare of Gifu University. Animal handling procedures were approved by the Committee for Animal Research and Welfare of Gifu University. Animal handling procedures were approved by the Committee for Animal Research and Welfare of Gifu University. ICR mice (Japan SLC Inc.) at 6-8 weeks of age were used with free access to food and water and were maintained on a $12 \mathrm{~h}$ light/dark cycle.

\subsection{Collection of embryos}

Female mice were superovulated with an intraperitoneal (ip) injection of 5 IU/animal of pregnant mare serum gonadotropin (PMSG) (Sigma Chemical Co, St. Louis, $\mathrm{MO})$ and ip injection of $5 \mathrm{IU} /$ animal of human chorionic gonadotropin (hCG) (Sigma Chemical Co.) at 48-hour intervals. For embryo collection, female mice were mated with males of proven fertility. Successful mating was determined the next morning by the presence of a vaginal plug which is defined as 0.5 day poscoitum $(0.5$ dpc). Animals were sacrificed by cervical dislocation. 8-cell stage embryos were collected early in the morning of $2.5 \mathrm{dpc}$ by flushing the oviducts. ICR female mice in estrous cycle were mated with vasectomized males of the same strain to be used as pseudo-pregnant recipient or foster mice for embryo transfer at $0.5 \mathrm{dpc}$ for oviduct transfer or $2.5 \mathrm{dpc}$ for uterine transfer.

\subsection{Generation of Col19a1nLacZ +/- mice}

Col19a1 5 and 3 homology arms were amplified using specific primers (Invitrogen) shown in table1 using PrimeStar Max polymerase (Takara, Japan), under the following conditions: $98^{\circ} \mathrm{C}$ for 30 sec, $98^{\circ} \mathrm{C}$ for $10 \mathrm{sec}, 55^{\circ} \mathrm{C}$ for $5 \mathrm{sec}$ and $72^{\circ} \mathrm{C}$ for $50 \mathrm{sec}$ for 20 cycles. These arms were subcloned into pMCS5 vector (MoBiTec) carrying Ampicillin resistant gene using Stbl3 Chemically Competent E. Coli (Invitrogen, Japan). The reporter gene $n L a c Z$ cassette was inserted in the first translation initiation site (ATG) in the $2^{\text {nd }}$ 
exon of Col19a1 locus inbetween the 5 and 3 homology arms. Col19a1 locus was targeted using CRISPR/Cas9 system in which the Cas9 induces double strand break (DSB) guided by gRNA sequence. The ES cells were transfected with the targeting construct and gRNA vector (pCAGGSU6gRNA-hCas9- PGK-Puro, Invitrogen) where the homologous recombination occurred as a DNA repair pathway. The ESCs clones were genotyped using genotyping primers shown in tablel by KAPA Taq Extra HotStar Polymerase, ReadyMix with dye (Kapabiosystems, Japan) under the following conditions: $95^{\circ} \mathrm{C}$ for $3 \mathrm{~min}, 95^{\circ} \mathrm{C}$ for $10 \mathrm{sec}, 55^{\circ} \mathrm{C}$ for $10 \mathrm{sec}$ and $72^{\circ} \mathrm{C}(1 \mathrm{~min} / 1 \mathrm{~kb})$ for 30 cycles. Chimeric mice were produced by microinjecting the positive ES cell clone in which the homologous recombination occurred into 8-cell stage embryo which then transplanted into pseudopregnant mice. We examined Col19a1 ${ }^{\text {nLacZ }}{ }^{+/}$mice from E14.5 to E18.5 using X-gal whole mount staining and immunohistochemical staining to detect $\beta$-galactosidase activity.

\subsection{X-gal staining}

For the whole-mount staining, mouse embryos or skin samples were collected from timed pregnant females in cool phosphate buffered saline (PBS). Tail biopsies were harvested for genotyping. Embryos were fixed in $4 \%$ paraformaldehyde (PFA, Wako) for 13 hours depending on the embryonic stage or $10 \mathrm{~min}$ for skin samples. After permeabilization by rinse buffer $(0.01 \%$ Na-deoxycholate (Wako), $0.02 \%$ NP40
(Wako), 2mM Mgcl2 (Wako) in PBS) for 3 times 10 minutes each, embryos were washed with PBS 3 times then stained in Xgal solution (5 mM K-ferricyanide (Wako), $5 \mathrm{mM}$ K-ferrocyanide (Wako), $2 \mathrm{mM}$ $\mathrm{MgCl} 2$ in PBS + X-gal (5-bromo-4-chloro3 -indolyl- $\beta$-D

-galactopyranoside $25 \mathrm{mg} / \mathrm{ml}$ Dimethyl formamide (Wako)) at $37^{\circ} \mathrm{C}$ overnight. The embryos were then post-fixed with $4 \%$ PFA. The embryos or skin samples were then cryoprotected with gradual series of sucrose (nacalai tesque) $15 \%, 30 \%$ in PBS at $4{ }^{\circ} \mathrm{C}$. The embryos were then embedded in Optimal Cutting Temperature (O.C.T.) medium (Tissue Tek, Sakura, Tokyo, Japan) and 6-8 $\mu \mathrm{m}$ cryosections were cut using a Cryostat (Leica CM1850, Germany) for H\&E staining.

\subsection{Immunohistochemical staining}

$8 \sim 10 \mu \mathrm{m}$ frozen sections were cut, completely dried, fixed in $4 \%$ PFA for $5 \sim 10$ min, rinsed with $0.05 \%$ Tween 20 (Wako)/PBS (PBS-T) 3 times 5 min each. Antigen retrieval was enhanced by incubating in $1 \%$ SDS (Sodium Lauryl sulphate (nacalai tesque)) in PBS-T for 5 min followed by washing in PBS-T for 5 min 3 times. The sections were blocked with 5\% Skim Milk (Wako) in PBS-T for 1 $\mathrm{hr}$ at $\mathrm{RT}$ or overnight at $4^{\circ} \mathrm{C}$ to reduce unspecific antibody binding. The sections were then incubated with primary antibodies diluted in blocking solution for 1 hr at RT or overnight at $4^{\circ} \mathrm{C}$ in moist chamber. Primary antibodies used were against $\beta$-galactosidase ( $\beta$-Gal, rabbit, Cappel, 1:1000), SOX2 (SRY (sex 
determining region Y-box2), rabbit, Invitrogen, 1:100) and Keratin 15 (K15, chicken, Polyclonal, Covance 1:500) followed by rinsing with PBS-T for $5 \mathrm{~min}$ three times at least. The tissue sections were then incubated with secondary antibodies (Alexa Fluor ${ }^{\circledR} 488$ and 555 donkey antirabbit $\operatorname{IgG}(\mathrm{H}+\mathrm{L})$ and Alexa Fluor ${ }^{\circledR} 594$ goat anti-chicken IgG $(\mathrm{H}+\mathrm{L})$, (Molecular Probes, Invitrogen)) diluted at 1:2000 in blocking solution together with DAPI (4', 6diamidino-2-phenylindole, dihydrochloride (Molecular Probes, Invitrogen)) at 1:1000 dilution to label the nuclei at RT for $1 \mathrm{hr}$. or $4^{\circ} \mathrm{C}$ overnight. Finally, the sections were washed 3 times in PBS-T and the slides were coverslipped with ProLong ${ }^{\circledR}$ Gold anti-fade reagent (Invitrogen).

\subsection{Imaging}

Gross images of whole mount stained embryos were obtained using a digital camera (Canon) or a dissection microscope (Leica MZ 16F) equipped with a camera (AxioCam MRc, Zeiss) driven by Axio Vision 4 software. Light and fluorescent microscopy images were obtained using a Keyence fluorescence microscope driven by BZ-II Viewer and Analyzer software.

Table 1: Primers used for PCR amplification and genotyping

\begin{tabular}{ll}
\hline \multicolumn{1}{c}{ Primer } & Sequence \\
\hline EcoRI_Col19a1_5Arm_FWD 5'-ACTGAATTCCTAAGGGGTGATACCTGGGAC-3' \\
KpnI_Col19a1_5Arm_REV & 5'-TGAGGTACCAACAAATGGCCACAGATCTGT-3' \\
MluI_Col19a1_3Arm_FWD & 5'-TGTACGCGTCAAGGCACAATGAGGCACACT-3' \\
AscI_Col19a1_3Arm_REV & 5'-TGAGGCGCGCCGACTGCTCTTTCAGAGGTT-3' \\
Col19a1_gRNA_FWD & 5'-CACCGCTCATTGTGCCTTGTAACCA-3' \\
Col19a1_gRNA_REV & 5'-AAACTGGTTACAAGGCACAATGAGC-3' \\
Col19a1_Genotyping1_FWD & 5'-AGCTAGCCTACTCCAAGCAGAGAAAGTGAG-3' \\
Col19a1_Genotyping1_REV & 5'-TGACTGTCAGACAGGTACATGCAGGAAGC-3' \\
Col19a1_Genotyping2_FWD & 5'-TGGATATCAATTGATTTCTCCCTGCTGGTG-3' \\
Col19a1_Genotyping2_REV & 5'-TAAATGCTCCAAATCAGCTGGAGAGATGGC-3' \\
nLacZ_Genotyping_FWD: & 5'-TTCTGTGTCTGCTGAGTTCCAGTTATCTGC-3' \\
nLacZ_Genotyping_REV: & 5'-CTGTTGAGCTGGGTCACTCCAGGGTTCTCC-3'
\end{tabular}

\section{RESULTS}

\subsection{Generation of a Col19a1LacZ allele}

Mouse Col19a1 has 3 splice variants or transcripts, the $1^{\text {st }}$ one contains 50 coding exons out of 51 exons, and the $2^{\text {nd }}$ has no coding exons out of 40 exons while the $3^{\text {rd }}$ contains 47 coding exons out of 48 exons. Among these exons, the first and second exons are shared by all of these splice variants. The First exon encodes only the $5^{\prime}$ 
untranslated sequence. Exon 2 contains part of $5^{\prime}$ the untranslated region. The Col19a $1^{\text {nlacz }}$ knock-in mice was generated by inserting $n L a c Z$ reporter gene at the first translation initiation site (ATG) of the $2^{\text {nd }}$ exon (Fig.1). After homologous recombination in ES cells, chimeric mice from correctly targeted ES clones were generated.

\subsection{Expression pattern of Col19a1 in mouse embryos}

To date, the hair development field has been lacking genetic drivers for specific targeting of DP cells, which are thought to interact with epithelial stem cells for morphogenesis to proceed. Here, we generated knock-in reporter mouse lines for specific expression in embryonic DP cells. In a series of wholemount X-Gal stained Col19a ${ }^{\text {LacZ }}$ embryos, we identified the expression of Col19a1 in the mouse embryonic skin (Fig. 2). Wide distribution of LacZ expression was detected in the hair follicles of the body, whiskers or vibrissae as well as the skin of leg and tail as shown in Fig.2. LacZ labeling was also detectable in the subcutaneous muscles and the external genitalia (data not shown).

\subsection{Expression pattern of Col19a1 during hair follicle morphogenesis}

The formation of HF is orchestrated by reciprocal interactions between the epidermis and the underlying mesenchyme. At E13.5, specialized dermal cells send the first signal to stem cells in the epidermis which rearrange to form hair placodes, which in turn back send a signal to the underlying dermal cells which aggregate to form dermal condensates or DP precursor cells. The DP precursor cells send again a signal to the hair placodes to initiate the proliferation and downgrowth of hair germs and pegs, with the DP cells in the lower edge. Matrix cells that reside at the base of the follicle bulb engulf the DP precursor cells to form the mature DP. This basic morphogenetic consequence of hair follicle formation is repeated in three separate waves giving rise to different hair follicle types.

To determine the stage point of Col19a $1^{\mathrm{LacZ}}$ expression in the dermal compartment of HF, we next analyzed sagittal embryo sections at different embryonic stages (Fig. 3 A-D). Col19a1 starts to be expressed in the dermal condensates or DP precursor cells of the hair germ stage (Fig. 3B, arrows). DP precursor cells continued to express $\mathrm{LacZ}$ in the down growing hair peg stage (Fig. 3C, arrow) and in the DP cells of mature HF (Fig. 3D, arrow) but it was not expressed in the dermal condensates of the early hair placode stage (Fig. 3A, arrow) at E14.5.

Col19a1 was expressed in the DP cells of the follicles of the three hair waves of E18.5 back skin (Fig. 4B). LacZ was sparsely expressed in the reticular layer of the dermis as well (Fig. 4B and 4C, arrow head). LacZ labeling was also detectable in the DP cells of $\mathrm{HF}$ of the ventral skin (Fig. 5B, arrow). Immunofluorescence staining confirmed the expression of Col19a1 by using anti- $\beta$ Gal and anti-Sox 2 antibodies. $\beta$-Gal was expressed in the DP cells of $1^{\text {st }}$ wave guard 
HF of back skin (Fig. 4C, arrow) and ventral skin (Fig. 5C, arrow). Follicles of other waves also showed expression of $\beta$-Gal (Fig. $4 C)$. $\beta$-Gal labeling was also detected in the reticular layer of the dermis of back skin (Fig. 4C, arrow head) and ventral skin (Fig. $5 \mathrm{C}$, arrow head). Sox 2 as a selectable DP marker was also expressed in the DP cells of HF of back skin (Fig. 4E, arrow) and ventral skin (Fig. 5E, arrow) confirming the expression of Col19a1 in the DP cells.

In the whiskers, Col19a1 was expressed in the ring sinus (Fig. 6C and 6D, arrow) and the neighboring part of inner conical body of whisker follicles. Interestingly, small whisker follicles in the periphery of the whisker showing lacZ labeling in the DP (Fig. 6D, arrow head) in addition to the ring sinus. Absence of lacZ expression in the DP of large whisker follicles up to E18.5 may be attributed to the late expression of Col19a1 in the whisker DP. Moreover, we found that the DP of hair follicles inbetween the whisker rows were also labeled with lacZ (Fig. 6C, arrow head). Immunohistochemical staining using anti$\beta$-Gal antibody confirmed the Col19a1 expression in the ring sinus of whisker (Fig. $6 \mathrm{E}$ and $6 \mathrm{~F}$, arrows) as well as the DP of small peripheral whisker follicles (Fig. 6F, arrow head).

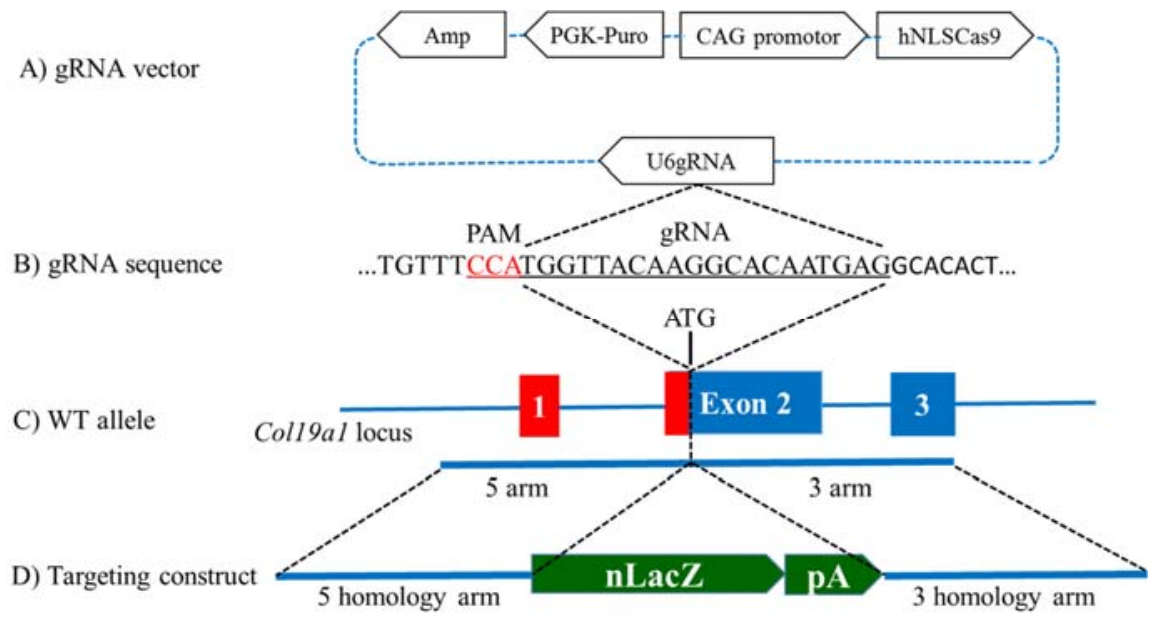

Fig. 1. Schematic representation of Col19a1 targeting strategy using CRISPR/Cas9 system in mice. A) gRNA vector containing Cas9 protein guided by guide RNA "gRNA"sequence. B) gRNA target sequence 20 nucleotides immediately upstream to Protospacer Adjacent Motif "PAM" indicated in red in the genomic target. Cas9 and gRNA will form a riboprotein complex "Cas9-gRNA complex" which will bind the site of genomic sequence with a PAM resulting in Double Strand Break "DSB" 3-4 nucleotides upstream of the PAM sequence. C) Wild-type allele in Col19a1 locus. D) Targeting construct includes homology arms (800 900bp) flanking $n L a c z$ reporter. $n L a c Z$ was inserted at the first translation initiation site "ATG" of $2^{\text {nd }}$ exon in Col19a1 locus. 

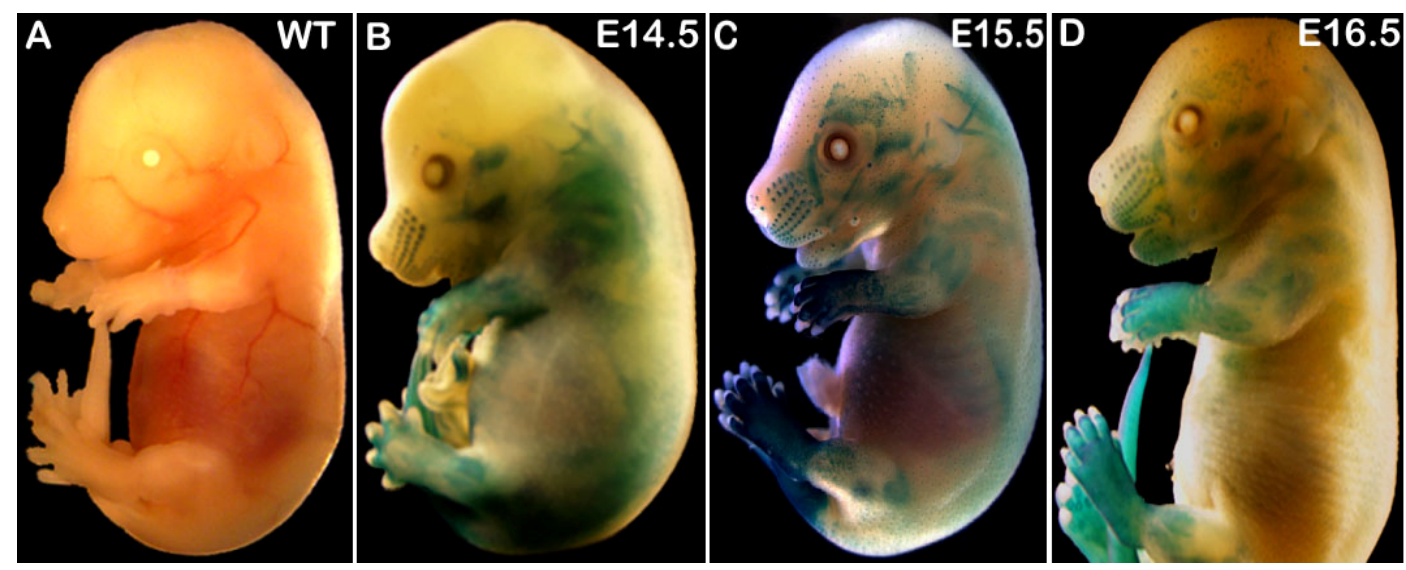

Fig. 2. Whole-mount X-Gal staining to show Col19a1 ${ }^{\text {LacZ }}$ expression in embryonic skin at E14.5 16.5.

A

E14.5 B

E15.5
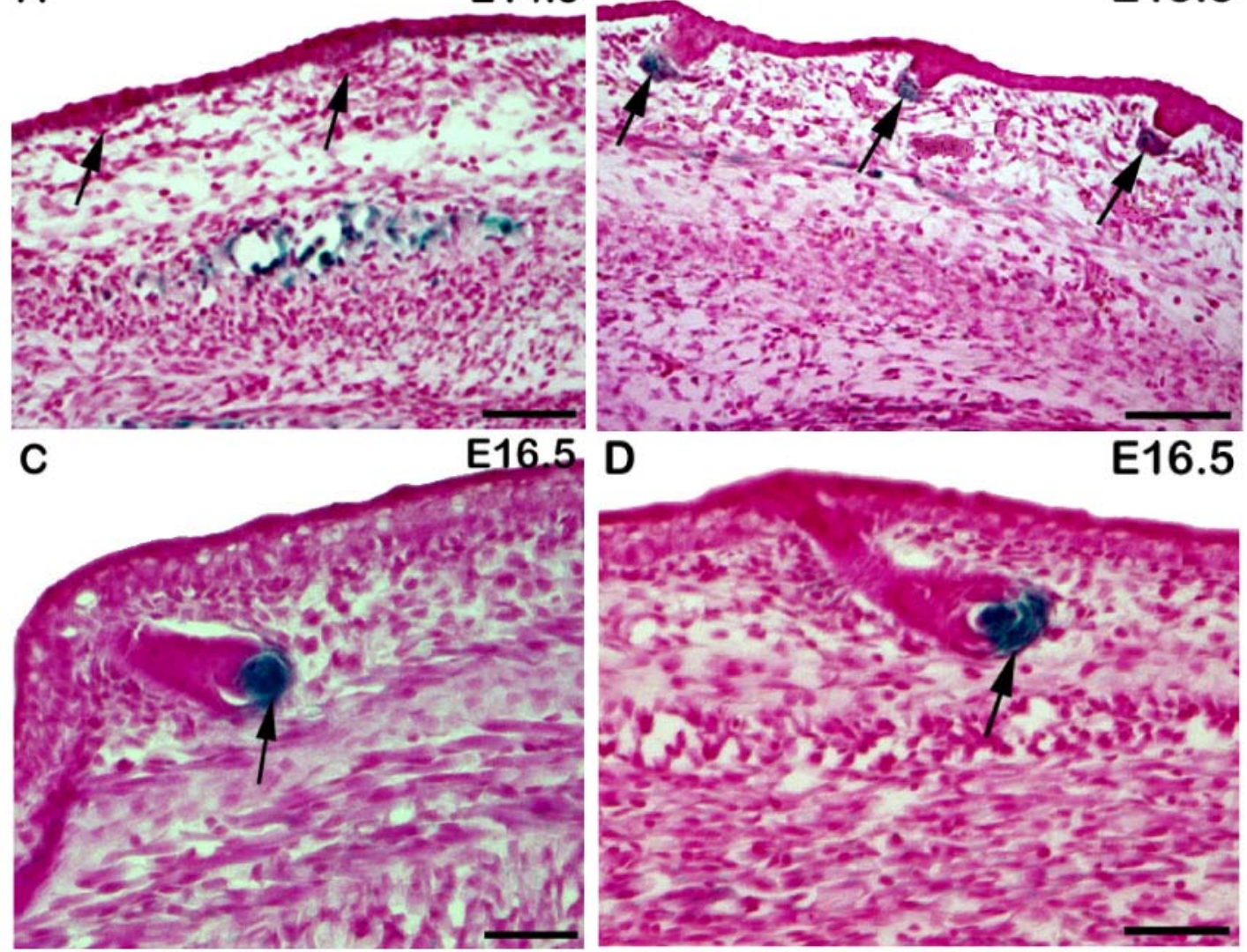

Fig. 3. Col19a1 expression pattern during HF morphogenesis by H\&E staining. A) Hair placode stage with no LacZ expression in the underlying dermal condensates or DP precursor cells (arrows) at E14.5. B) Hair germ stage showing LacZ expression in the dermal condensates or DP precursor cells (arrows) at E15.5. C) Hair peg stage showing LacZ expression in the DP cells (arrow) at E16.5. D) Mature $1^{\text {st }}$ wave HF showing LacZ expression in the DP cells (arrow) at E16.5. Scale bars, $50 \mu \mathrm{m}$ (A-D). 

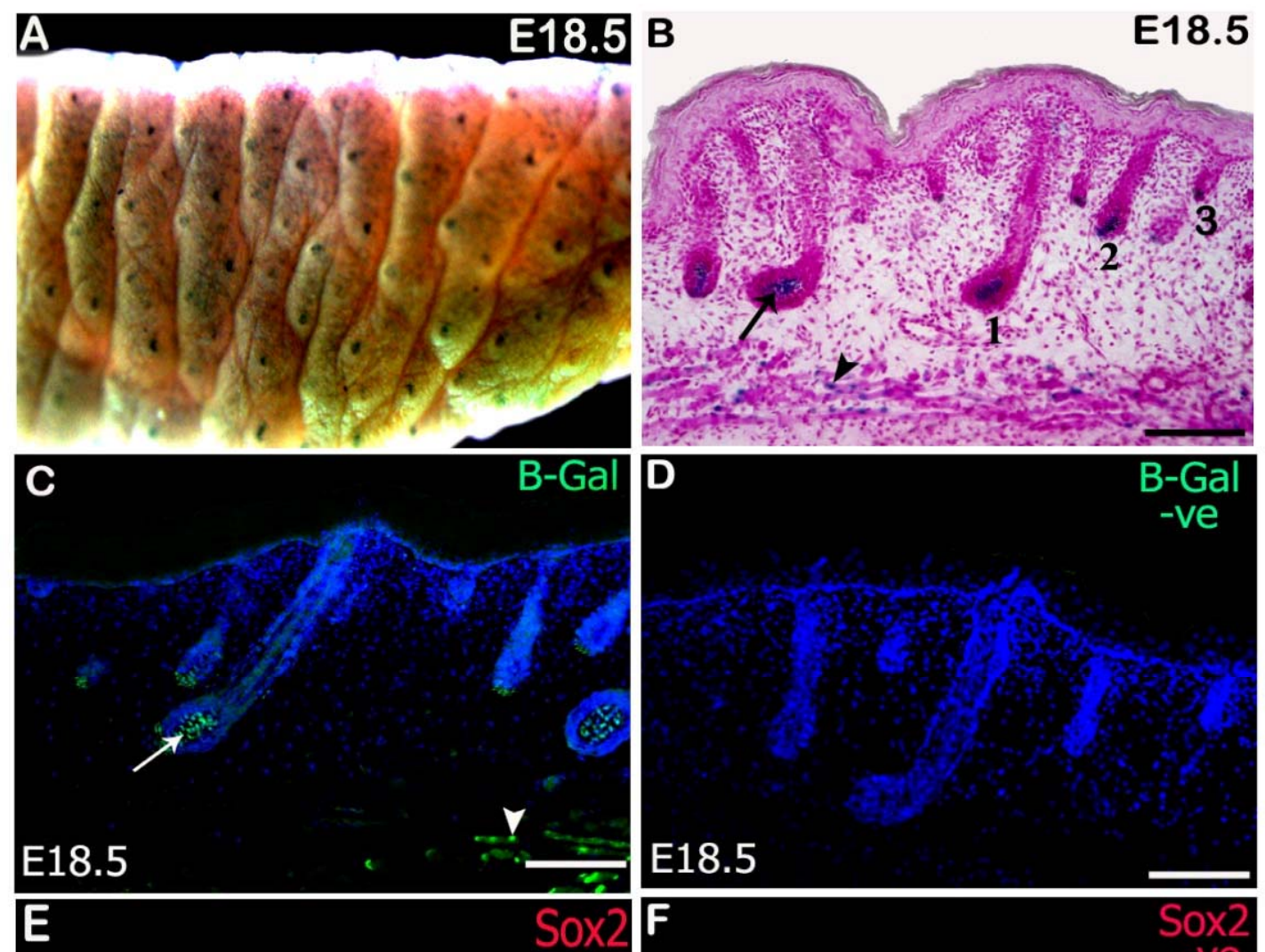

E18.5

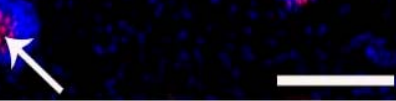

E18.5

Fig. 4. Col19a1 expression in the back skin of E18.5 knock-in reporter mouse embryos. A) Whole mount stained back skin showing lacZ labeling in HF. B) H\&E staining of back skin showing lacZ labeling in DP cells (arrow) in the follicles of the 3 different hair waves indicated by numbers. LacZ labeling was also detectable in the dermis indicated by arrow head. (C-F) Immunohistochemical staining. C) LacZ expression identified by anti- $\beta$-galactosidase ( $\beta$-Gal) in the DP cells (arrow) and in the dermis (arrow head). D) Negative control for anti- $\beta$-Gal. E) Sox2 expression in the DP cells (arrow). F) Negative control for anti-Sox2. Nuclei were counterstained with DAPI (blue). Scale bars, $50 \mu \mathrm{m}$ (B-F). 


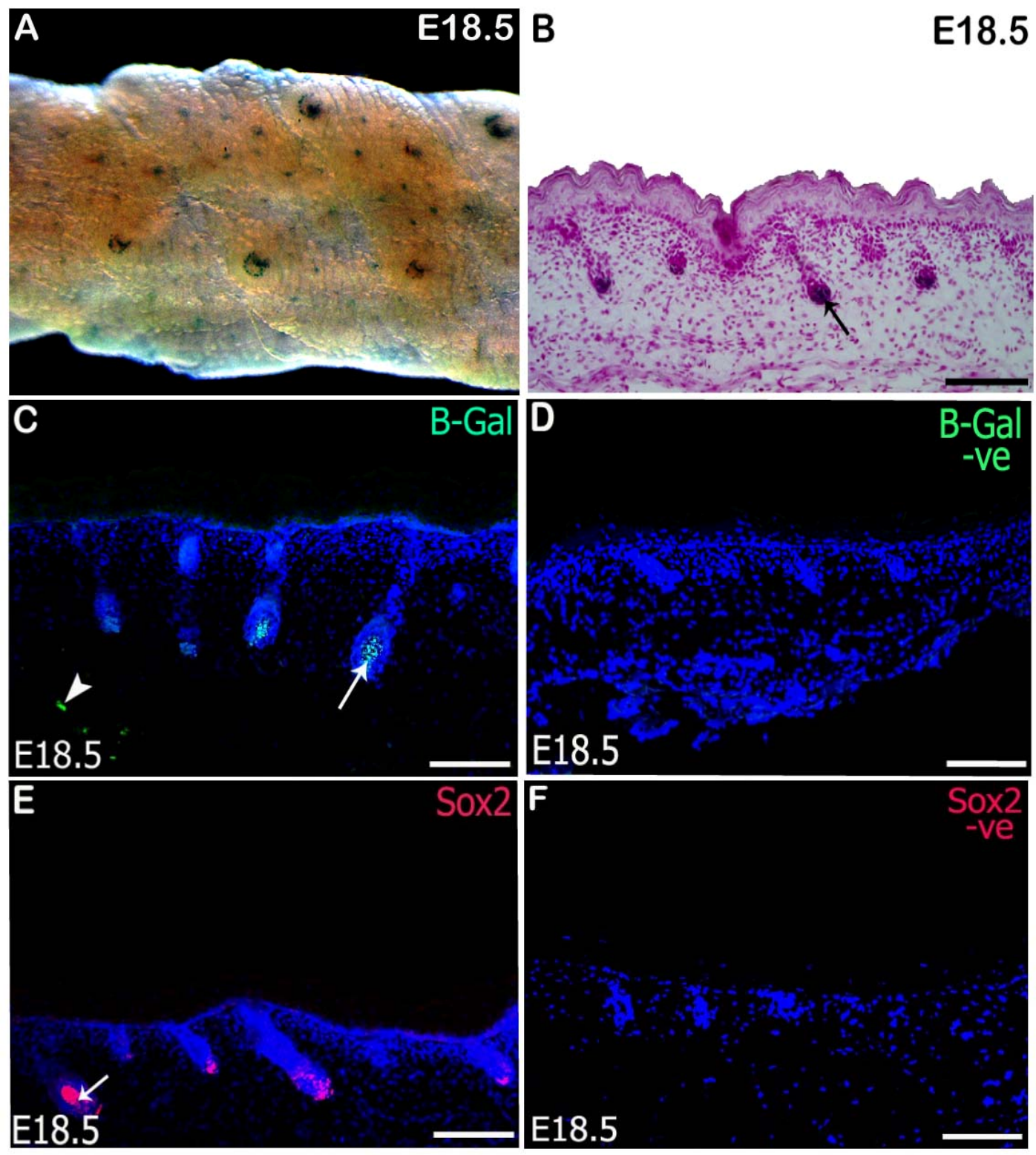

Fig. 5. Col19a1 expression in the ventral skin of E18.5 knock-in reporter mouse embryos. A) Whole mount stained ventral skin showing lacZ labeling in HF. B) H\&E staining of ventral skin showing lacZ labeling in DP cells (arrow). (C-F) Immunohistochemical staining. C) LacZ expression identified by anti- $\beta$-galactosidase ( $\beta$-Gal) in the DP cells (arrow) and in the dermis (arrow head). D) Negative control for anti- $\beta$-Gal. E) Sox2 expression in the DP cells (arrow). F) Negative control for anti-Sox2. Nuclei were counterstained with DAPI (blue). Scale bars, $50 \mu \mathrm{m}(\mathrm{B}-\mathrm{F})$. 


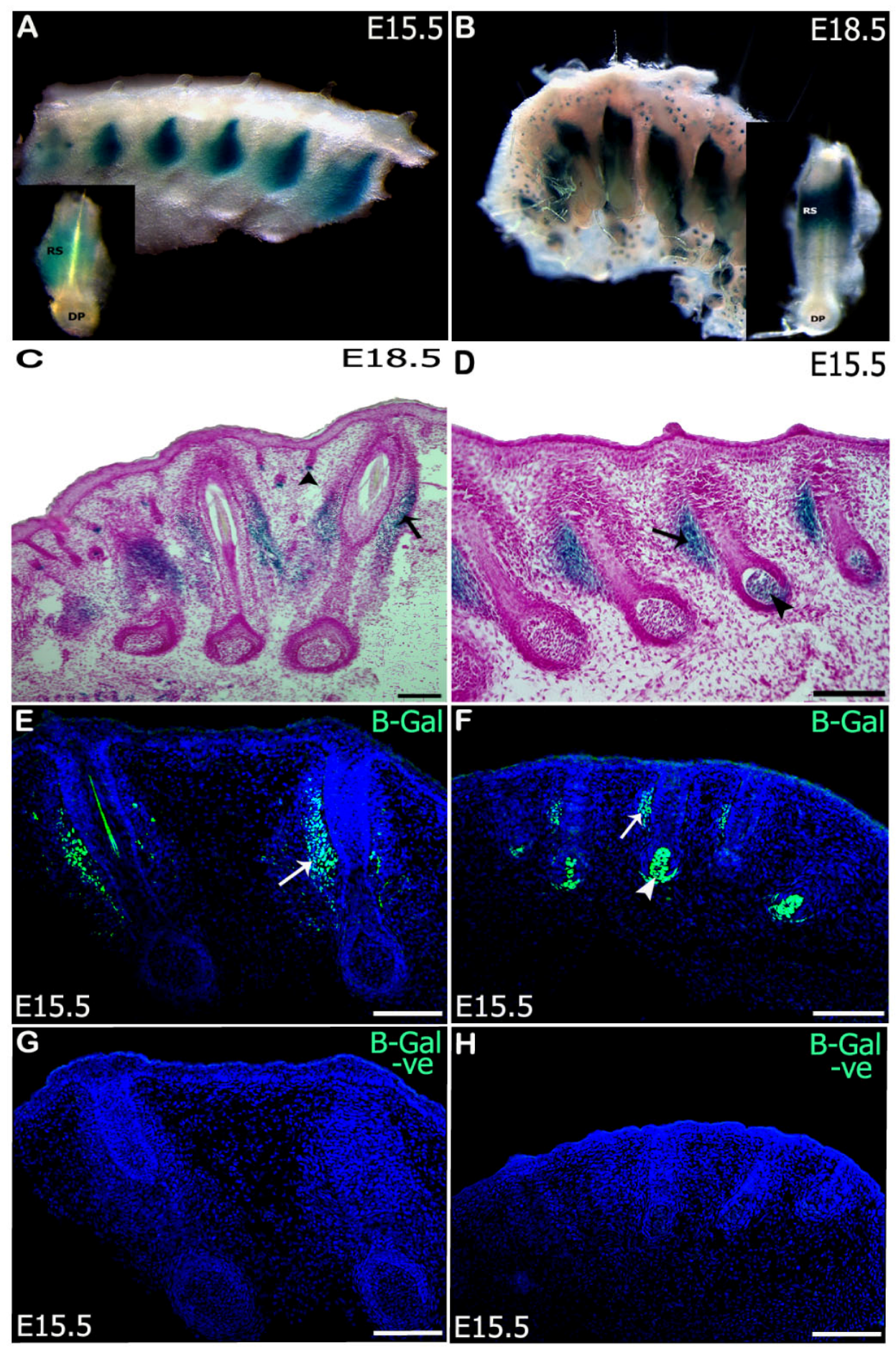

Fig. 6. Col19a1 expression in the whiskers of lacZ knock-in mouse embryos. A, B) Whole mount stained whisker of E15.5 and E18.5 embryos respectively. Insert is single whisker follicle showing lacZ labeling in the ring sinus (RS) and the neighboring part of inner conical body. C) H\&E staining of whisker at E18.5 showing lacZ labeling in the ring sinus (arrow). LacZ labeling was also detectable in the DP (arrow head) of small hair follicles inbetween the whisker follicles. D) H\&E staining of whisker at E15.5 
showing lacZ labeling in the ring sinus (arrow). LacZ labeling was also detectable in the DP (arrow head) of small whisker follicles at the periphery of the whisker. (E-H) Immunohistochemical staining. E, F) LacZ expression identified by anti- $\beta$-galactosid- ase ( $\beta$-Gal) in the ring sinus (arrow) and in the DP of small peripheral whisker follicles (arrow head). G, H) Negative control for anti- $\beta$-Gal. Nuclei were counterstained with DAPI (blue). Scale bars, $50 \mu \mathrm{m}(\mathrm{C}-\mathrm{H})$.

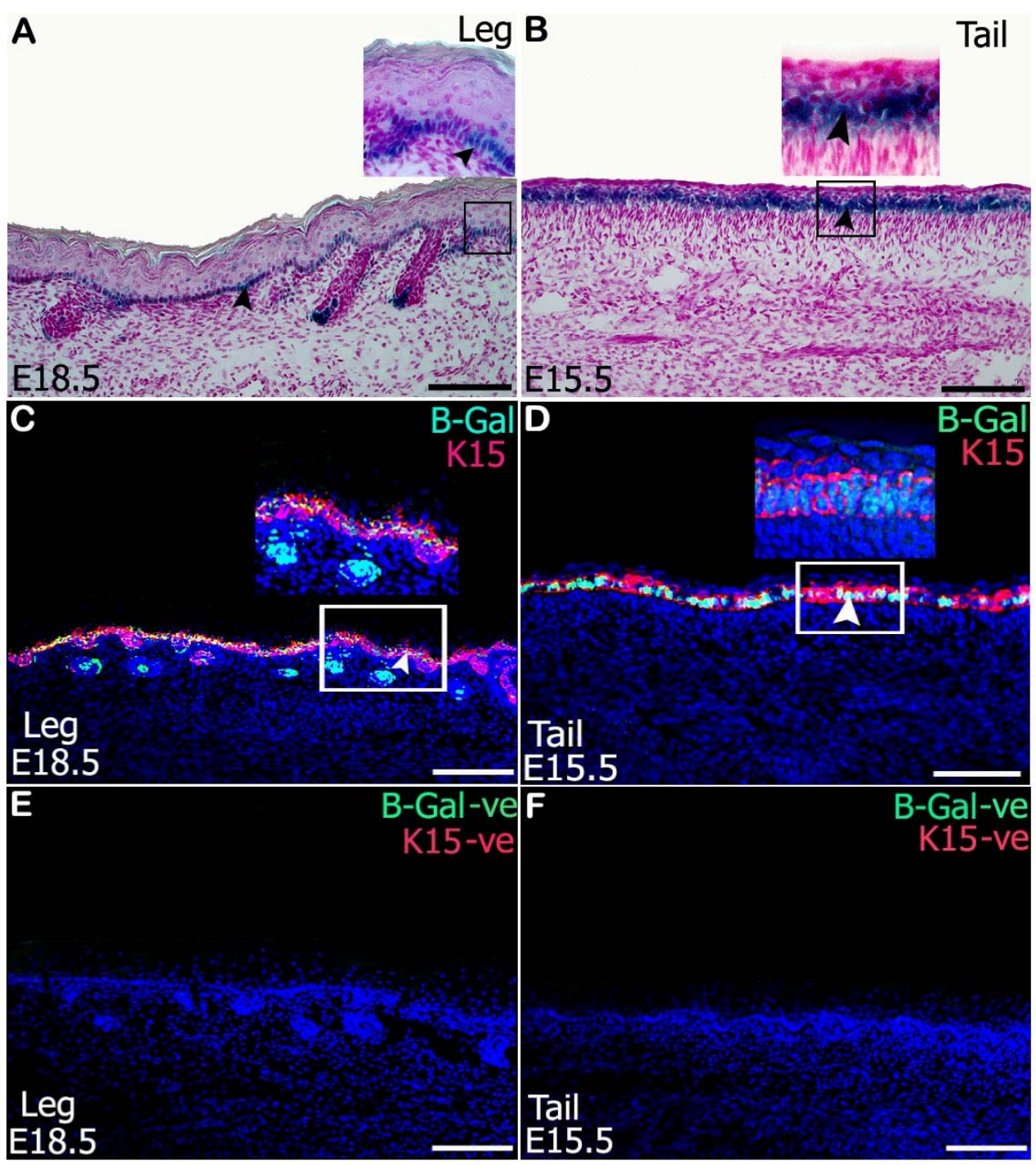

Fig. 7. Col19a1 expression in the skin of leg and tail of lacZ knock-in mouse embryos. A, B) H\&E staining of E18.5 leg skin and E15.5 tail respectively showing lacZ labeling in the basal keratinocyte layer of the epidermis (arrow head). Insert is higher magnification. (C-F) Immunohistochemical staining. C, D) Double staining for leg skin and tail respectively using anti- $\beta$-Gal and anti-K15 showing lacZ expression in the basal keratinocyte layer of the epidermis (arrow head). Insert is higher magnification. E, F) Negative control for double stained leg and tail respectively. Nuclei were counterstained with DAPI (blue). Scale bars, $50 \mu \mathrm{m}(\mathrm{A}-\mathrm{F})$. 


\subsection{Dermal-epidermal expression of Col19a1 in the leg and tail skin}

Surprisingly, in addition to expression of Col19a1 in the DP cells of HF of leg and tail skin, it was also expressed in the basal keratinocyte layer of epidermis (Fig. 7A and 7B) of leg and tail respectively. Immunolabeling by double staining using anti- $\beta$-Gal and anti-K15 antibodies confirmed the expression of Col19al in the leg and tail skin. $\beta-G a l$ was expressed in the basal keratinocyte layer of the epidermis where the keratinocyte epidermal marker K15 expressed in the leg (Fig. 7C, arrow head) and tail (Fig. 7D, arrow head). This result confirmed the epidermal expression of Col19a1 in the leg and tail skin.

\section{DISCUSSION}

The present study declared that Col19a1 starts to be expressed in the mouse embryonic skin and dermal condensates or DP cells at E14.5 but it was detected in the skin of E16.5 embryos (Sumiyoshi et al., 2001). The latter authors added that Col19a1 expression during embryogenesis commences at $\sim \mathrm{E} 9.5$ in the myotome and with a pattern that closely follows the myogenic regulatory factor myf-5. On the other hand, Sumiyoshi et al. (1997) stated that Col19a1 transcripts can be detected as early as 11 days of gestation and in all embryonic tissues, except the liver, of an 18 dpc mouse. In contrast, CD133 as a DP marker starts to be expressed by the DP cells at E16.5 (Ito et al., 2007) while Corin is first detected in the dermal condensate at E15 (Enshell-Seijffers et al., 2008). Sox2 starts to be detected in the dermal condensates at E14.5 (Driskell et al., 2009) similar to Col19a1 in the present study.

Our results showed that Col19a1 was detected in the dermis as sparse amount in the reticular layer. This result was in contrast with Myers et al. (1997) who stated that the dermis was nonreactive with Col19 antibody.

In contrary to some DP markers like Tbx 18 which is expressed in the dermal condensates of the earliest placode stage of HF (Grisanti et al., 2013), the current study showed that Col19a1 ${ }^{\text {LacZ }}$ started to be expressed in the dermal condensates of hair germ stage of HF not the hair placode. Therefore, it is tempting to speculate that Col19a1 expression is not mediated by the first wave of hair follicle morphogenesis, but rather than induced by the consequence of the second wave of epidermal and dermal interactions.

In conclusion, the results of the current study are in an agreement with the previous notion that Col19a1 is expressed in the skin and muscles of mouse embryos (Sumiyoshi et al., 1997). The major new finding reported here is the discovery that Col19a1 was also expressed in the ring sinus and the adjacent part of inner conical body of whiskers and the DP cells of HF starting from hair germ stage as well as in the basal keratinocyte layer of leg and tail epidermis. Meanwhile, Sumiyoshi et al. (2001) declared that Col19a1 is expressed preferentially and transiently in differentiating muscles cells as well as smooth muscle layers of the esophagus, 
stomach and the forming adductory muscles around the hair follicles. We hypothesize that Col19a1 may be involved in signaling pathways required for cell migration and differentiation during mesenchymalepithelial interaction for HF morphogenesis. Future studies will help in functional assessment of Col19a1 during $\mathrm{HF}$ development.

From the expression of Col19a1 in the basal keratinocyte layer of epidermis of limbs and tail, we can predict that Col19a1 plays a fundamental role in the keratinization of thick skin in the limbs and tail.

Col19-null mice are normal at birth but, $\sim 95 \%$ of pups die within the first 3 weeks, presumably caused by their inability to feed due to defects in muscle development in the lower esophageal sphincter demonstrating a role for Col19a1 in the development of skeletal muscle transdifferentiation in the mouse esophagus (Sumiyoshi et al., 2004). A secondary phenotype has also been observed in hippocampal neurons in mice lacking Col19. Neuronal morphology is normal; however, some subtypes of hippocampal synapses were malformed, demonstrating a role for Col19 in the nervous system (Su et al., 2010). These mouse studies suggest that Col19a1 may be playing multiple functions during development.

Surprisingly, Mammalian ECM including collagens, derived from various tissues and organs, has been used as a biologic scaffold for therapeutic regenerative applications (Badylak, 2005). More interestingly, DP cells can be reprogrammed into induced pluripotent stem (iPS) cells that can express pluripotency genes and differentiate into cells from all germ layers in vitro and contribute to chimeric mice in vivo, including the germline (Tsai et al., 2010). This opens avenue for using skin derived iPS cells in regenerative medicine.

Ongoing generation of Col19a1 null mice will ultimately explore the functional role of Col19a1 during HF morphogenesis. From this perspective, it is plausible to expect that future studies will provide additional functional evidence for Col19a1 which might be used as a powerful tool for elucidating the mechanism of $\mathrm{HF}$ development.

\section{ACKNOWLEDGMENTS}

This work was supported by grants to Prof. Masatake Osawa from Massachusetts General Hospital, Harvard Medical School, NIAMS, JSPS, National Cancer Institute and Graduate School of Medicine of Gifu University.

\section{REFERENCES:}

Badylak, S.F. 2005. Regenerative Medicine and Developmental Biology: The Role of the Extracellular Matrix. The Anatomical Record (Part B: New Anat.) 287B:36 - 41.

Driskell, R.R., Giangreco, A., Jensen, K.B., Mulder, K.W. and Watt, F.M. 2009. Sox2-positive dermal papilla cells specify hair follicle type in mammalian epidermis. Development, 136, 2815-2823. 
Enshell-Seijffers, D., Lindon, C. and Morgan, B.A. 2008. The serine protease Corin is a novel modifier of the agouti pathway. Development 135, 217-225.

Grisanti, L., Clavel, C., Cai, X., Rezza, A., Tsai, S., Sennett, R., Mumau, M., Cai, C. and Rendl, M. 2013. Tbx18 Targets Dermal Condensates for Labeling, Isolation, and Gene Ablation during Embryonic Hair Follicle Formation. Journal of Investigative Dermatology, vol. 133, 344-353.

Ito, Y., Hamazaki, T.S., Ohnuma, K., Tamaki, K., Asashima, M. and Okochi, H. 2007.

Isolation of Murine Hair-Inducing Cells Using the Cell Surface Marker Prominin-1/CD133. Journal of Investigative Dermatology, 127, 1052-1060.

Khaleduzzaman, M., Sumiyoshi, H., Ueki, Y., Inoguchi, K., Ninomiya, Y., and Yoshioka, H. 1997. Structure of the human type XIX collagen (COL19A1) gene, which suggests it has arisen from an ancestor gene of the FACIT family. Genomics 45:304-312.

Myers, J.C., Yang, H., D’Ippolito, J.A., Presente, A., Miller, M.K., Dion, A.S. 1994. The triple-helical region of human type XIX collagen consists of multiple collagenous subdomains and exhibits limited sequence homology to $\alpha 1(\mathrm{XVI})$. J Biol Chem 269:1854918557.
Myers, J.C., Li, D., Bageris, A., Abraham, V., Dion, A.S., and Amenta. P.S. 1997. Biochemical and immunohistochemical characterization of human type XIX defines a novel class of basement membrane zone collagens. Am. J. Pathol. 151:1729-1740.

Myers, J.C., Li, D., Amenta, P.S., Clark, C.C., Nagaswami, C., and Wiesel, J.W. 2003. Type XIX collagen purified from human umbilical cord is characterized by multiple sharp kinks delineating collagenous subdomains and by intermolecular aggregates via globular, disulfide-linked and heparin-binding amino termini. J. Biol. Chem. 278:32047-32057.

Myllyharju, J., and Kivirikko, K.I. 2004. Collagens, modifying enzymes and their mutations in humans, flies and worms. Trends Genet. 20:33-43.

Oudart, J.B., Brassart-Pasco, S., Vautrin, A., Sellier, Ch., Machado, C., DupontDeshorgue, A., Brassart, B., Baud, S., Dauchez, M., Monboisse, J.C., Harakat, D., Maquart, F.X., and Ramont, L. 2015. Plasmin releases the anti-tumor peptide from the $\mathrm{NC1}$ domain of collagen XIX. Oncotarget, 6(6): $3656-3668$.

Ramirez, F., and Rifkin, D. 2003. Cell signaling events: a view from the matrix. Matrix Biol. 22:101-107.

Ribatti, D. and Santoiemma, M. 2014. Epithelial-mesenchymal interactions: a fundamental Developmental Biology mechanism. Int. J. Dev. Biol. 
58: 303-306.

Sennett, R. and Rendl, M. 2012. Mesenchymal-epithelial interactions during hair follicle morphogenesis and cycling. Semin Cell Dev Biol. 23(8): 917-927.

$\mathrm{Su}$, J., Gorse, K., Ramirez, F. and Fox, M.

A. 2010. Collagen XIX is expressed by interneurons and contributes to the formation of hippocampal synapses. $J$. Comp. Neurol. 518, 229-253.

Sumiyoshi, H., Inoguchi, K., Khaleduzzaman, M., Ninomiya,Y., Yoshioka, H. 1997. Ubiquitous expression of the $\alpha 1$ (XIX) collagen gene (Col19a1) during mouse embryogenesis becomes restricted to a few tissues in the adult organism. $\mathrm{J}$ Biol Chem 272:17104-17111.

Sumiyoshi, H., Laub, F., Yoshioka, H., and Ramirez, F. 2001. Embryonic expression of type XIX Collagen is transient and confined to Muscle Cells. Developmental Dynamics
220:155-162.

Sumiyoshi, H., Mor, N., Lee, S.Y., Doty, S., Henderson, S., Tanaka, S., Yoshioka, H., Rattan, S., and Ramirez, F. 2004. Esophageal muscle physiology and morphogenesis require assembly of a collagen XIX-rich basement membrane zone. J. Cell Biol. 166, 591-600.

Thein, M.C., McCormack, G., Winter, A.D., Johnstone, I.L., Shoemaker, C.B. and Page, A.P. 2003. Caenorhabditis elegans Exoskeleton Collagen COL19: An Adult-Specific Marker for Collagen Modification and Assembly, and the Analysis of Organismal Morphology. Developmental Dynamics, 226:523-539.

Tsai, S., Clavel, C., Kim, S., Ang, Y., Grisanti, L., Lee, D., Kelley, K. and Rendl, M. 2010. Oct4 and Klf4 Reprogram Dermal Papilla Cells into Induced Pluripotent Stem Cells. STEM CELLS; 28:221-228. 